\title{
ZIGBEE WIRELESS NETWORK-BASED INDOOR MOBILE ROBOT MONITORING AND POSITIONING METHOD UNDER BINOCULAR VISION
}

\author{
Xiaowei Chen', Quoc Tien Le ${ }^{2}$, Rd Rohmat Saedudin ${ }^{3}$ \\ 1Shangqiu Polytechnic Henanshangqiu, 476000, China \\ ${ }^{2}$ Haiphong Department of Education and Training, Haiphong, Vietnam \\ ${ }^{3}$ School of Industrial Engineering, Telkom University, 40257, Bandung, West Java, Indonesia \\ Email: xweichen00471@163.com
}

\begin{abstract}
Accuracy of the positioning system can be improved by a low-cost and practical ZigBee wireless network indoor mobile robot monitoring and positioning system. Based on the application requirements, the software design and hardware design of the indoor mobile robot are carried out to build an intelligent mobile robot system independently. Under the binocular vision system, the Received Signal Strength Indication (RSSI) ranging technology is adopted as the ZigBee positioning algorithm. The deficiencies of the traditional RSSI algorithm are improved, and the system is tested in the hall corridors and rooms. In the indoor rooms, the mean relative error (MRE) of the RSSI algorithm before the improvement is 23.27\%, and the MRE of the RSSI algorithm after the improvement is $13.91 \%$, with an increase of $9.36 \%$. In the hall corridor, the MRE of the RSSI algorithm before the improvement is $14.86 \%$, and the MRE of the RSSI algorithm after the improvement is $10.82 \%$, with an increase of $4.04 \%$. The improved RSSI algorithm can meet the monitoring and positioning requirements of indoor mobile robot based on ZigBee wireless network under binocular vision.
\end{abstract}

Keywords: Binocular Vision, ZigBee Wireless Network, Indoor Mobile Robot, Monitoring and Positioning, RSSI Algorithm.

\section{Introduction}

With the continuous development and progress of science and technology, the field of mobile robots has become one of the most active research hot spots [1]. For mobile robots, positioning technology is very critical. The lack of a positioning function means that the mobile robot cannot move autonomously, identify a position, and achieve real intelligence. Traditional robot positioning methods mainly include Global Positioning System (GPS), wheel odometer, and inertial navigation system. These methods can independently complete simple positioning functions; however, they also have the disadvantages of positioning errors and difficulty in indoor positioning [2]. Machine vision can enhance the robot's ability to sense the environment and has the advantages of low power consumption, complete information acquisition, and wide detection range [3]. According to the number of cameras in the system, machine vision technology can be divided into three types, i.e., monocular, binocular, and multi-ocular. Binocular vision can obtain the information of the object through multiple angles, and it can recover the three-dimensional (3D) information of the object according to the geometric relationship, which has a wide range of applications [4]. Therefore, this study will conduct research based on binocular vision. To further improve the positioning accuracy of indoor mobile robots, only relying on binocular vision is far from enough. Loganathan et al. (2019) applied ZigBee wireless positioning technology to indoor mobile robots [5]. ZigBee wireless network, as one of the key technologies of the Internet of Things (IoT), has the characteristics of energy-saving, low cost, close range, low speed, and short delay. Due to these characteristics, it can be used for information transmission between network devices with large node density, wide distribution range, low rate, and low energy consumption [6].

In summary, although ZigBee wireless network applications and chip shipments are increasing annually, in general, ZigBee wireless networks are still in the initial exploration stage. Positioning technology has prodigious significance for mobile robots. To improve the positioning accuracy of indoor mobile robots, this study combines binocular vision systems and ZigBee wireless networks to propose an optimized positioning algorithm for monitoring and positioning of indoor mobile robots. Thereby achieving the positioning in indoor 
environments with multiple precisions, low power consumption, low cost, and flexible installation and maintenance. The innovation of this study lies in combining the binocular vision system and research on ZigBee wireless network-based indoor mobile robot monitoring and positioning algorithm to guide indoor mobile robot positioning.

\section{Method}

\subsection{Overall design of the indoor mobile} robot monitoring and positioning system

The indoor mobile robot monitoring and positioning system based on ZigBee wireless network is a visual wireless positioning system composed of a positioning monitoring center and a wireless positioning network. The functional block diagram of the system is shown in Figure 1. It includes collecting reference node data, calculating position coordinates of mobile nodes, wireless transmission, optimizing positioning data accuracy, and displaying positioning data.

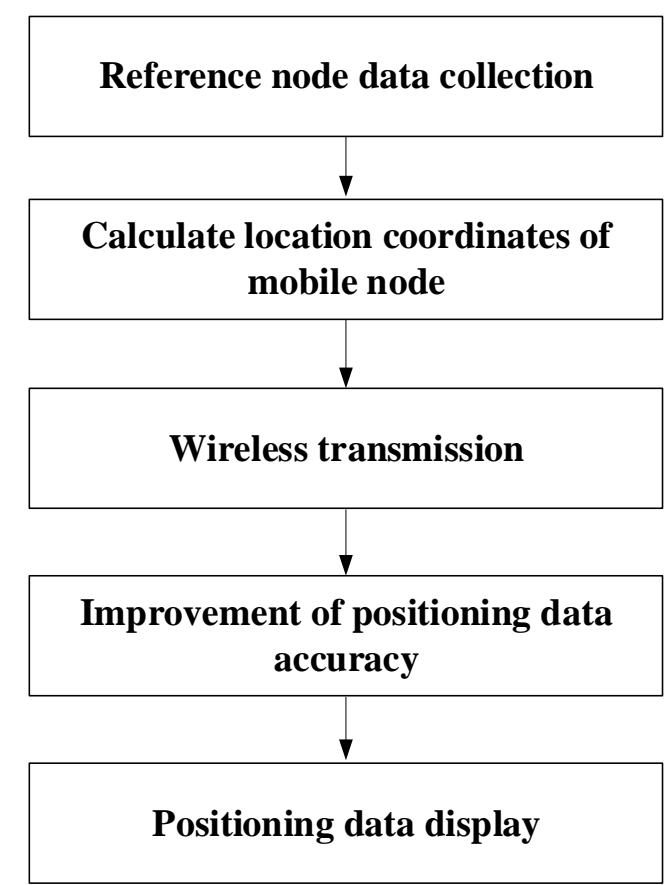

Figure 1: Functional block diagram of the indoor mobile robot monitoring and positioning system

Based on the application requirements, the embedded technology is selected for the software design and hardware design of the indoor mobile robot, and the intelligent mobile robot system is built independently. The overall hardware structure of the robot includes a perception layer, a computing layer, a control layer, and a mechanical layer. The relationship among the layers is shown in Figure 2. The sensing layer, the computing layer, and the control layer transmit information through a serial port connection and are installed on the mechanical layer.

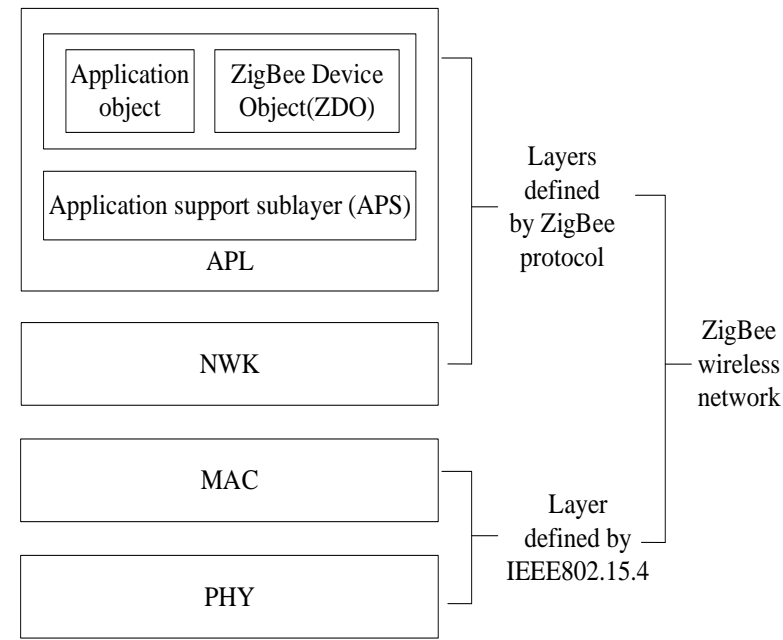

Figure 2: Overall hardware structure of the robot

The system hardware includes a Personal Computer (PC) running Linux operating system, an indoor mobile robot, a binocular camera, six ZigBeebased network nodes, a router, a wireless Universal Serial Bus (USB), an Advanced RISC Machine (ARM) development board, and various cables. Comprehensively considering the performance and cost, the CC2430/31EM chip is selected as the chip for the ZigBee positioning system. This chip has the advantages of simple structure, energy-saving, and efficient operation, and it complies with the IEEE802.15.4 standard [7].

To realize the indoor mobile robot monitoring and positioning system based on ZigBee wireless network, it is necessary to ensure that the system has good processing performance, network communication capabilities, low power consumption, and stable and smooth operation. ARM11 has the ARM V6 instruction set architecture, which has low cost, low power consumption, and excellent operating system performance. Therefore, this study chooses ARM11 as the embedded processor. The S3C6410 is designed based on the ARM11 core. It has built-in powerful hardware accelerators such as audio and video processing, two-dimensional (2D) acceleration, and display scaling. It also integrates Multi-Format video Code (MFC) and has powerful video processing capabilities. The wireless network includes a wireless network adapter and a wireless router, which can complete the transmission of positioning data between the PC and the ARM. The router selected in this study is the TP-LINK150M wireless broadband router, which has the characteristics of stable data transmission and reception and is compatible with $11 \mathrm{~b} / \mathrm{g} / \mathrm{n}$ wireless network adapters.

The software design is divided into monitoring software (upper computer software), driver software, and lower computer software. The software development environment is IAR Embedded Workbench (EW) software (Texas 
Instruments). EW is currently the most popular embedded development tool. It integrates multiple functions and can provide a unified user interface for different processors, which is simple and efficient [8]. In addition, EW is highly flexible and can develop application software for different microcontrollers and microprocessors.
The layered structure of the ZigBee protocol stack is shown in Figure 3. The physical (PHY) layer and the media access control (MAC) layer at the bottom are the layers defined by the IEEE802.15.4 specification and the application (APL) layer and network (NWK) layer are norms and standards established by the ZigBee Alliance.

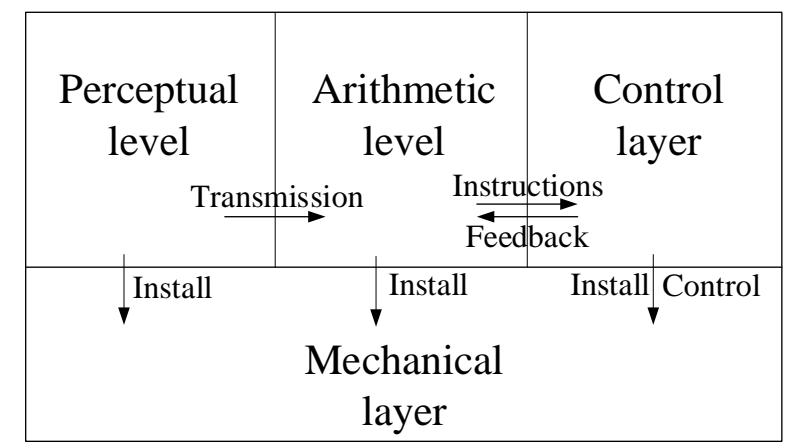

Figure 3: Hierarchical structure of ZigBee protocol stack

The flowchart of system initialization is shown in Figure 4 . The system initialization is to complete the various environments required to initialize the hardware and software architecture and to prepare for the operation of the operating system; also, when the system completes initialization, it will enter a low-power sleep mode.

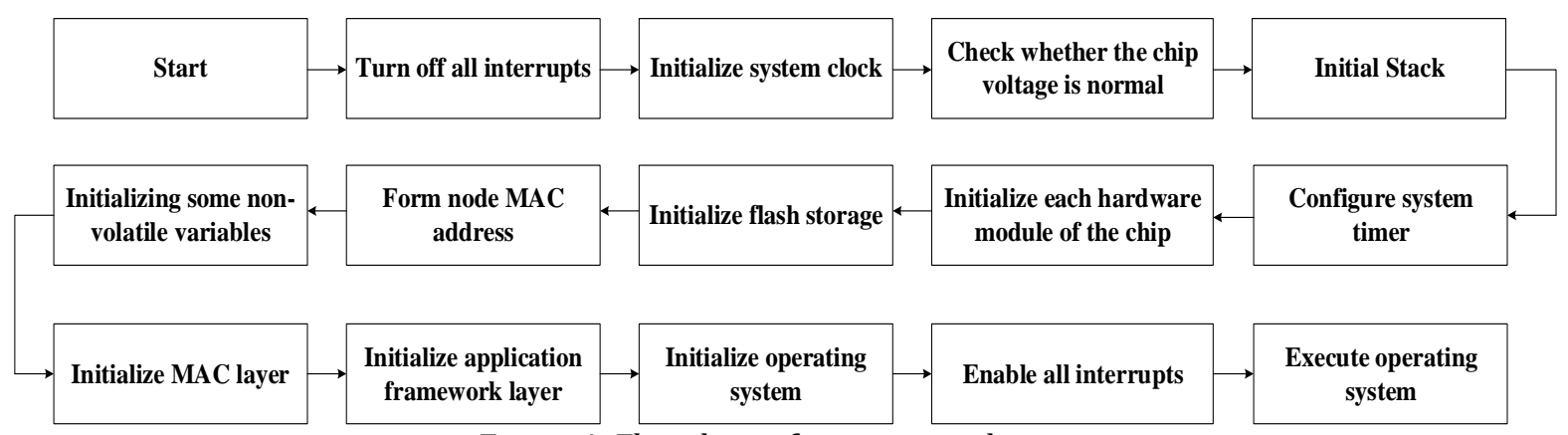

Figure 4: Flowchart of system initialization

There is no network adapter driver in the ARMLinux system used when the ARM development board system is transplanted. Therefore, the wireless network adapter driver transplantation needs to be performed. The NW336 USB network adapter (Shenzhen NETAC, China) is selected as the key device in the wireless transmission in the system. When configuring the kernel, before recompiling and transplanting, the chip is supported first. After starting the target board, the system support for the chip can be found from the serial debugging information.

\subsection{Binocular vision system}

This vision system is introduced for indoor mobile robots. Two or more cameras are used to obtain image information from different positions on the same object point, and simultaneously recover distance information from parallax based on the triangulation principle.
First, the camera calibration is performed to determine the internal and external parameters of the camera [9]. When any point on the image is known as the image pixel coordinates, the coordinates of the actual point corresponding to the point on the world coordinate system cannot be calculated. Therefore, two parallel-aligned binocular cameras need to be configured to capture unknown points at different angles, thereby obtaining two rays. These two rays are located on the same plane, and the intersection point is the exact position of the unknown point in the 3D scene. At this time, the triangulation method is used to calculate the unknown point to obtain the actual position of the unknown point. The principle of the binocular vision system is shown in Figure 5. The world coordinate system and the camera coordinate system are coincident. The $(\mathrm{u} 0, \mathrm{v} 0)$ point is the image center point, and (u1, v1) and (u2, v2) are respective projection points of the object point $\mathrm{P}$ in the 3D scene on the left and right image pixel coordinate systems. 


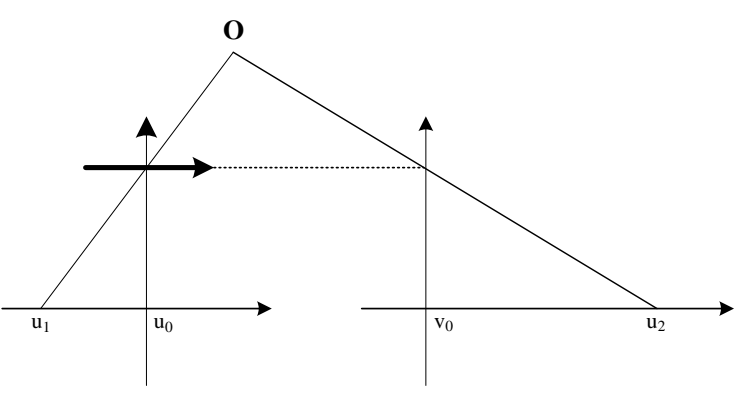

Figure 5: Schematic diagram of binocular vision system

\subsection{ZigBee positioning algorithm}

In the research of wireless positioning networks, the positioning algorithm is the core, whose pros and cons directly affect the level of node positioning accuracy. This study uses Received Signal Strength Indication (RSSI) ranging technology as the ZigBee positioning algorithm. RSSI ranging technology is a low-cost and easy-to-implement ranging algorithm. Combining the signal power of the transmitting node with the received power of the mobile node, this algorithm measures the distance of a node based on the attenuation of the radio signal as the transmission distance increases. In the case of measuring multiple distances, the trilateration method is used for positioning calculation [10]. However, in practical applications, signal strength loss is often caused by obstacles, poor antenna characteristics, and other factors; thus, the distance error is large. Therefore, based on the RSSI algorithm, this study improves the RSSI algorithm according to the changes in indoor spatial signals, as well as the positioning accuracy of the algorithm.

The signal model of the RSSI algorithm is shown in Equation (1).

$$
R S S I=-(10 \times n \times \lg d+A)
$$

Where: $\mathrm{n}$ represents the attenuation value of the signal strength which increases by $1 \mathrm{~m}$ every time the signal travels, $d$ represents the distance between the positioning node and the base station, and A represents the absolute value of the received energy intensity at a distance of $1 \mathrm{~m}$ from the transmitter.

However, in practical applications, the positioning node is mobile, and only a model cannot determine the attenuation degree of the signal received by the node. On the one hand, the attenuation factor of the signal will change as the blocking object changes. On the other hand, the position of the blocking object in the position space between the mobile node and the reference node will also change as the position of the mobile node changes. In the RSSI algorithm, the trilateration method is shown in Figure 6. In the ideal case (Figure 6A), by using the distance between the positioning node and the three reference nodes (d, e, f) as the radius, three circles D, E, F are made.
The three circles intersect at one point, and the intersection point is the coordinate $\mathrm{O}$ of the moving node. However, in the actual situation (Figure 6B), blocking objects and noise will attenuate the signal. There are three intersections $(a, b, c)$ between the three circles, and they do not intersect at one point. Thus, the resulting mobile node positioning accuracy is poor.

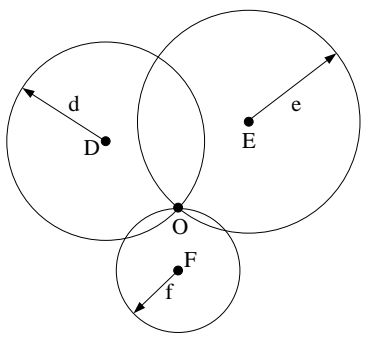

A

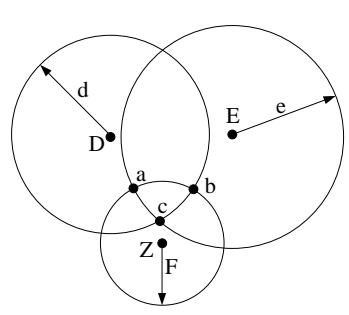

B
Figure 6: Trilateral measurement method (A: ideal situation; B: actual situation)

To improve the RSSI algorithm, first, the measurement distance between the mobile node and the reference node is corrected by the least square method, and the error caused by the environmental impact is compensated [11]. The distance between the moving node $(\mathrm{X}, \mathrm{Y})$ and each reference node $(\mathrm{Xi}$, $\mathrm{Yi}$ ) is as shown in Equation (2).

$x_{i}=\sqrt{\left(X_{i}-X\right)^{2}+\left(Y_{i}-Y\right)^{2}}, i=1,2,3,4$

Combining Equation (1) and least squares, the sum of squared deviations is assumed as in Equation (3).

$$
P(a, b)=\sum_{i=1}^{n}\left(a x_{i}+b-y_{i}\right)^{2}
$$

Where yi represents the distance from the mobile node to the i-th reference node.

The relationship between the corrected distance and the estimated distance is deduced as Equation (4).

$$
y^{\prime}=a \times \frac{-R S S I-A}{10 n}+b
$$

The three intersections of the three circles form an overlapping area. To calculate the coordinate values of the mobile nodes, this study uses the centroid positioning algorithm to calculate the average of the three intersections to make up for the limitations of the trilateration method. The coordinates of three reference nodes that are not on the same straight line are defined as (xa, ya), (xb, yb), and $(\mathrm{xc}, \mathrm{yc})$.

The coordinates of the mobile node are derived from the distance between the two points as Equation (5). 


$$
\left[\begin{array}{l}
x \\
y
\end{array}\right]=\left[\begin{array}{l}
2\left(x_{1}-x_{3}\right) 2\left(y_{1}-y_{3}\right) \\
2\left(x_{2}-x_{3}\right) 2\left(y_{2}-y_{3}\right)
\end{array}\right]^{-1}\left[\begin{array}{c}
x_{1}^{2}-x_{3}^{2}+y_{1}^{2}-y_{3}^{2}+r_{3}^{2}-r_{1}^{2} \\
x_{1}^{2}-x_{3}^{2}+y_{2}^{2}-y_{3}^{2}+r_{3}^{2}-r_{2}^{2}
\end{array}\right]
$$

Where: ra, rb, and rc respectively represent the distances between the mobile node and the three reference nodes.

Considering the positioning accuracy and cost comprehensively, 4 reference nodes are selected to participate in the positioning measurement [12]. To avoid accidental errors, 10 RSSIs are read and the average after removing a minimum and a maximum is obtained.

\subsection{Positioning system test}

When testing the positioning system, the parameters are measured first. In the RSSI algorithm, the parameter $\mathrm{A}$ of the mobile node (a signal strength value at $1 \mathrm{~m}$ from the transmitting end) and the parameter $\mathrm{n}$ (a signal strength attenuation value of $1 \mathrm{~m}$ away from the transmitting end) have an important effect on the positioning accuracy. The parameter $A$ is related to the hardware, and the $\mathrm{n}$ parameter is related to the actual environment. To reduce the systematic error caused by parameter $A$ and parameter $n$, the experimental model is improved by introducing error factors. In the ideal case, the parameter $A$ is equal in any direction. However, the influence of the antenna on the transmitter and receiver is not the same as the parameter A in actual measurement. In this case, the error can be reduced by averaging multiple measurements. Figure 7 is a schematic diagram of the actual measurement of parameter A. The RSSI value of the signal transmitted by the central node $\mathrm{O}$ is measured at points $\mathrm{A}, \mathrm{B}, \mathrm{C}$, and $\mathrm{D}$, and the average value is taken [13-17].

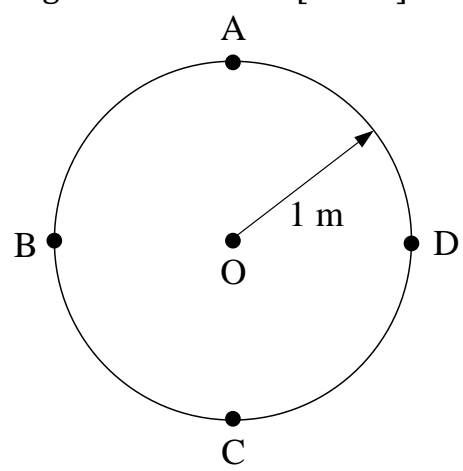

Figure 7: The schematic diagram of actual measurement of parameter $A$

To reduce the complexity of hardware implementation, the CC2431 positioning engine uses the indirect value $n$ sub instead of the parameter $n$. The value range of $n_{-}$sub is $0-31$. Measurement is performed under the same environment, and the signal strengths are measured at the distances d1 and $\mathrm{d} 2$ from the transmitting end, respectively. The parameter $n$ is calculated by Equation (6).

$$
n=\frac{R S S I_{2}-R S S I_{1}}{101 g\left(\frac{d_{1}}{d_{2}}\right)}
$$

Where: RSSI 1 is the signal strength at $\mathrm{d} 1$ from the transmitter, RSSI 2 is the signal strength at $\mathrm{d} 2$ from the transmitter.

Then, the parameter $\mathrm{n}$ is converted to $\mathrm{n} \_$sub. At the same time, errors are reduced by averaging multiple measurements.

In the buildings, the main space is the rooms and the hall corridor. Therefore, the test is performed in the hall corridor and the rooms respectively, and the monitoring and positioning results before and after improvement are compared.

\section{Results and Discussion}

\subsection{Monitoring and positioning results of indoor rooms}

The monitoring and positioning results of the indoor room are shown in Table 1 and Figure 8. Compared with that before the improvement, the accuracy of mobile robot monitoring and positioning in the indoor room after the improvement has been significantly enhanced. Before the improvement, the mean relative error (MRE) of the RSSI algorithm positioning is $23.27 \%$. After the improvement, the MRE of the RSSI algorithm positioning is $13.91 \%$, with an increase of $9.36 \%$. It can be found in the figure that the positioning coordinates measured by the improved RSSI algorithm show extreme instability, it is difficult to determine the positioning, and the coordinate deviation is large. Therefore, it is related to influencing factors such as electromagnetic interference from indoor computers, air conditioners, people walking around, and blocking objects. Therefore, the improved RSSI algorithm cannot be applied to indoor positioning, where the electromagnetic environment is complex and blocking objects are various. The estimated coordinates of the improved RSSI algorithm are closer to the actual coordinates. They have higher stability, which greatly reduces the MRE of positioning and improves the accuracy of monitoring and positioning. It shows that the improved RSSI algorithm can be better applied to indoor positioning, where the electromagnetic environment is complex with various blocking objects. 
Table 1: Monitoring and positioning results of indoor rooms

\begin{tabular}{|c|c|c|}
\hline Actual coordinates $(\mathrm{X}, \mathrm{Y}) / \mathrm{m}$ & $\begin{array}{c}\text { Estimated coordinates before } \\
\text { improvement }(\mathrm{X}, \mathrm{Y}) / \mathrm{m}\end{array}$ & $\begin{array}{c}\text { Estimated coordinates after } \\
\text { improvement }(\mathrm{X}, \mathrm{Y}) / \mathrm{m}\end{array}$ \\
\hline$(1.00,10.00)$ & $(1.20,9.50)$ & $(1.20,9.80)$ \\
\hline$(2.00,9.00)$ & $(0.70,9.60)$ & $(2.50,9.40)$ \\
\hline$(3.00,8.00)$ & $(1.10,8.10)$ & $(2.80,8.40)$ \\
\hline$(4.00,7.00)$ & $(4.50,6.00)$ & $(5.50,6.70)$ \\
\hline$(5.00,6.00)$ & $(7.00,7.00)$ & $(5.60,4.00)$ \\
\hline$(6.00,5.00)$ & $(4.30,5.30)$ & $(7.30,4.60)$ \\
\hline$(7.00,4.00)$ & $(6.00,4.50)$ & $(8.00,3.60)$ \\
\hline$(8.00,3.00)$ & $(8.80,5.00)$ & $(9.10,2.70)$ \\
\hline$(9.00,2.00)$ & $(8.20,1.20)$ & $(9.60,2.00)$ \\
\hline$(10.00,1.00)$ & $(9.50,3.00)$ & \\
\hline
\end{tabular}

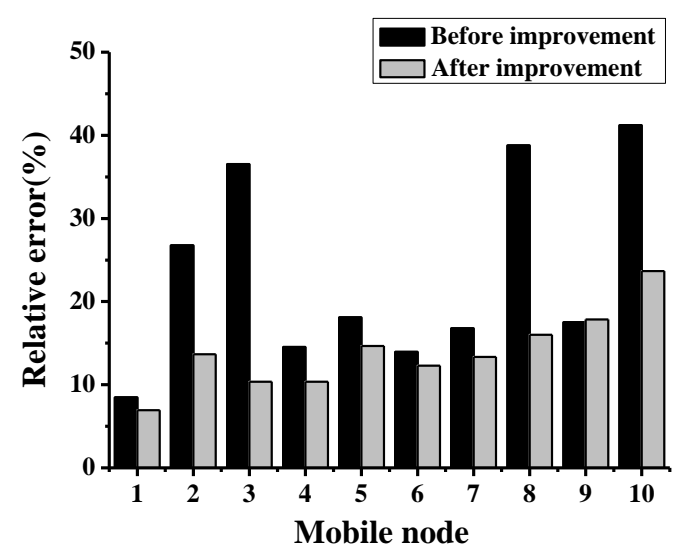

Figure 8: MRE of indoor room monitoring positioning before and after improvement

\subsection{Monitoring and positioning results of the hall corridor}

The monitoring and positioning results of the hall corridor are shown in Table 2 and Figure 9.
Compared with that before the improvement, the accuracy of the mobile robot monitoring and positioning in the hall corridor after the improvement has been significantly enhanced. Before the improvement, the MRE of the RSSI algorithm positioning is $14.86 \%$. After the improvement, the MRE of the RSSI algorithm positioning is $10.82 \%$, with an increase of $4.04 \%$.

Compared with indoor room monitoring and positioning, the MRE of the hall corridor monitoring and positioning is lower before the improvement. So, it is for the reason that compared with indoor rooms, the hall corridor is wider and less obstructed. However, the RSSI algorithm before the improvement is still affected by the random error of the measurement and the multi-path effect of the signal.

Also, the improved RSSI algorithm can overcome these shortcomings and obtain higher monitoring and positioning accuracy. Therefore, the improved RSSI algorithm can significantly improve the accuracy of monitoring and positioning, whether in indoor rooms or hall corridors.

Table 2: Monitoring and positioning results of the hall corridor

\begin{tabular}{|c|c|c|}
\hline $\begin{array}{c}\text { Actual coordinates }(\mathrm{X}, \\
\mathrm{Y}) / \mathrm{m}\end{array}$ & $\begin{array}{c}\text { Estimated coordinates before improvement } \\
(\mathrm{X}, \mathrm{Y}) / \mathrm{m}\end{array}$ & $\begin{array}{c}\text { Estimated coordinates after } \\
\text { improvement }(\mathrm{X}, \mathrm{Y}) / \mathrm{m}\end{array}$ \\
\hline$(1.00,1.00)$ & $(1.90,0.40)$ & $(1.50,0.50)$ \\
\hline$(2.00,2.00)$ & $(2.20,1.00)$ & $(2.20,1.50)$ \\
\hline$(3.00,3.00)$ & $(2.50,4.00)$ & $(2.50,3.10)$ \\
\hline$(4.00,4.00)$ & $(4.50,4.50)$ & $(5.00,4.25)$ \\
\hline$(5.00,5.00)$ & $(5.00,4.00)$ & $(6.50,6.00)$ \\
\hline$(6.00,5.00)$ & $(6.70,6.90)$ & $(8.50,3.50)$ \\
\hline$(8.00,4.00)$ & $(9.00,3.00)$ & $(10.00,4.00)$ \\
\hline$(10.00,3.00)$ & $(10.20,4.10)$ & $(11.60,1.90)$ \\
\hline$(12.00,2.00)$ & $(11.00,1.90)$ & $(13.00,1.50)$ \\
\hline$(14.00,1.00)$ & $(13.00,2.00)$ & \\
\hline
\end{tabular}




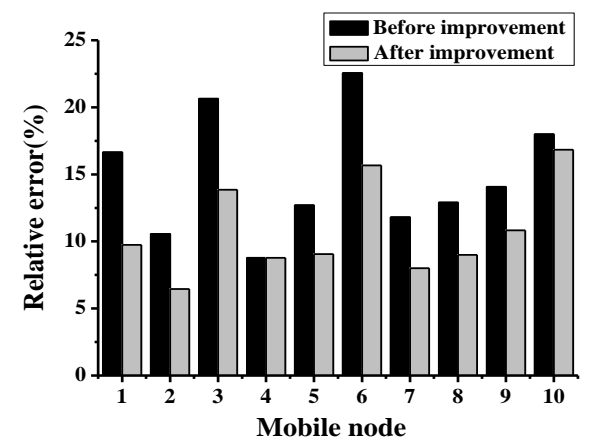

Figure 9: MRE of hall corridor monitoring positioning before and after improvement

\section{Conclusions}

To improve the monitoring and positioning accuracy of indoor mobile robots, this study uses ZigBee wireless network as the core to carry out software design and hardware design under the binocular vision system. The traditional RSSI algorithm is improved to build a ZigBee wireless network indoor mobile robot monitoring and positioning system. System testing shows that the improved RSSI algorithm can significantly improve the accuracy of monitoring and positioning, whether in indoor rooms or hall corridors. Also, it can meet the monitoring and positioning requirements for indoor mobile robots. The analysis and improvement of the wireless sensor positioning algorithm based on ZigBee technology in this study have important practical application significance. However, there are also deficiencies in the research process. The positioning algorithm used in this study is based on 2D space expansion, and the actual environment is 3D. Therefore, in the subsequent research process, the algorithm will be extended to 3D space to obtain more valuable results.

\section{References}

[1] Fan Q, Sun B, Sun Y, et al. Data fusion for indoor mobile robot positioning based on tightly coupled INS/UWB[J]. The Journal of Navigation, 2017, 70(5), pp. 1079-1097.

[2] Aykaç M, Erçelebi E, Aldin N B. ZigBee-based indoor localization system with the personal dynamic positioning method and modified particle filter estimation[J]. Analog Integrated Circuits and Signal Processing, 2017, 92(2), pp. 263-279.

[3] Yu X, Fan Z, Wan $H$, et al. Positioning, Navigation, and Book Accessing/Returning in an Autonomous Library Robot using Integrated Binocular Vision and QR Code Identification Systems[J]. Sensors, 2019, 19(4), pp. 783.

[4] Zhang Z, Guo W X, Su L, et al. Stereo Matching Algorithm for Robot Binocular Images in Real Time[J]. Beijing Ligong Daxue Xuebao/Transaction of Beijing Institute of Technology, 2017, 37(9), pp.975-981.
[5] Loganathan A, Ahmad N S, Goh P. Self-Adaptive Filtering Approach for Improved Indoor Localization of a Mobile Node with ZigbeeBased RSSI and Odometry[J]. Sensors, 2019, 19(21), pp.4748.

[6] Luo R C, Hsiao T J. Dynamic Wireless Indoor Localization Incorporating With an Autonomous Mobile Robot Based on an Adaptive Signal Model Fingerprinting Approach[J]. IEEE Transactions on Industrial Electronics, 2018, 66(3), pp. 1940-1951.

[7] S. Sathishkumar, Dr. M. Kannan. Topology Optimization of Integrated Combustion Engine Piston Using $\mathrm{F}$ ea Method (Cae Tools). Acta Mechanica Malaysia, 2019, 2(1): 01-05.

[8] L. Natrayan, E. Aravindaraj, M.S. Santhosh, M. Senthil Kumar. Analysis and Optimization of Connecting Tie Rod Assembly In Agriculture Application. Acta Mechanica Malaysia, 2019, 3(1): 06-10.

[9] Syed Mamun R Rasid, Md. Belayet Hossain, Md. Emdadul Hoque, Md. Ariful Azam Arif And Md. Sarikat Ali Sarder. Modeling and Control of a Magnetic Levitation System Using an Analog Controller. Acta Electronica Malaysia, 2019, 3(2): 41-44.

[10] Ahmed M. Alturas, Abdulmajed O. Elbkosh, Othman Imrayed. Stability Analysis of DC-DC Buck Converters. Acta Electronica Malaysia, 2020, 4(1): 01-06.

[11] Neelam Gupta, Neel Kanth. Analytical Approximate Solution Of Heat Conduction Equation Using New Homotopy Perturbation Method. Matrix Science Mathematic, 2019, 3(2): 01-07.

[12] Jiansheng P, Jian M, Qingjin W E I, et al. An indoor mobile robot positioning system based on radio-frequency identification[J]. Journal Europeen des Systemes Automatises, 2017, 50(3), pp. 313.

[13] Bianchi V, Ciampolini P, De Munari I. RSSI-based indoor localization and identification for ZigBee wireless sensor networks in smart homes[J]. IEEE Transactions on Instrumentation and Measurement, 2018, 68(2), pp. 566-575.

[14] Zhao M, Li H, Tong Y. Research on the calibration method of "bi-binocular" stereo vision system[J]. Guangxue Jishu/Optical Technique, 2017, 43(5), pp. 385-393.

[15] Coşgun E, Korkmaz H, Toker K. An Embedded System Design to Build Real-Time 2D Maps for Unknown Indoor Environments[J]. Sakarya Üniversitesi Fen Bilimleri Enstitüsü Dergisi, 2019, 23(4), pp. 617-632.

[16] Murthy C R G, Patil R, Dixit S S, et al. Implementation of an Indoor Navigation Robot for Visually Impaired and New Visitors[J]. iManager's Journal on Embedded Systems, 2017, 6(1), pp. 6.

[17] Lau L, Quan Y, Wan J, et al. An autonomous ultra-wide band-based attitude and position determination technique for indoor mobile laser scanning[J]. ISPRS International Journal of Geo-Information, 2018, 7(4), pp. 155. 